\title{
Fish Recognition Model for Fraud Prevention using Convolutional Neural Networks
}

\author{
Rhayane S. Monteiro • Morgana C. O. \\ Ribeiro - Calebi A. S. Viana - Mário W. \\ L. Moreira • Glácio S. Araújo • Joel J. \\ P. C. Rodrigues
}

Received: date / Accepted: date

\begin{abstract}
Fraud, misidentification, and adulteration of food, whether unintentional or purposeful, are a worldwide and growing concern. Aquaculture and fisheries are recognized as one of the sectors most vulnerable to food fraud. Besides, a series of risks related to health and distrust between consumer and popular market that this sector develop an effective solution for fraud control. Species identification is an essential aspect to expose commercial fraud. Convolutional neural networks $(\mathrm{CNNs})$ are one of the most powerful tools for image recognition and classification tasks. Thus, the objective of this study is to propose a model of recognition of fish species based on CNNs. The results obtained show an algorithm with an accuracy of $86 \%$, providing an effective solution to prevent fish fraud.
\end{abstract}

\section{R. Monteiro}

Federal Institute of Education, Science, and Technology of Ceará (IFCE)

E-mail: rhayane.monteiro@ifce.edu.br

M. Ribeiro

Federal Institute of Education, Science, and Technology of Ceará (IFCE)

E-mail: morgana.cassia.oliveira05@aluno.ifce.edu.br

C. Viana

Federal Institute of Education, Science, and Technology of Ceará (IFCE)

E-mail: calebi.assis.silva03@aluno.ifce.edu.br

M. Moreira (Corresponding author)

Federal Institute of Education, Science, and Technology of Ceará (IFCE)

E-mail: mario.wedney@ifce.edu.br

G. Araújo

Federal Institute of Education, Science, and Technology of Ceará (IFCE)

E-mail: glacio@ifce.edu.br

J. Rodrigues

Federal University of Piauí (UFPI)

Instituto de Telecomunicações (IT/Portugal)

E-mail: joeljr@ieee.org 
Keywords Food fraud · Fish recognition · Computer vision · Convolutional neural networks

\section{Introduction}

According to the International Featured Standards (IFS) guide ${ }^{1}$ the deliberate and intentional substitution, incorrect labeling, adulteration or falsification of food, raw materials, ingredients, or packaging is defined as product fraud. The food fraud, in turn, is a growing global concern [1]. With globalization and complex distribution systems, food fraud has become commonplace, with far-reaching impact and adverse consequences on the well-being of the population. According to the Food Integrity Manual, prepared by Food Integrity ${ }^{2}$, adulteration is the most common, considering the types of food fraud. However, food authentication covers many other topics, such as geographic origin, variety identification, production method, as well as the detection of genetically modified organisms. According to Song et. al, food fraud is a problem that has a significant impact on individuals, companies, and society and can therefore cause a public health risk, business loss, and reduced consumer confidence in brands [2].

Fish and other seafood are one of the most commercialized products in the world [1]. According to Probst, due to its performance on a global scale, the fishing sector is frequently challenged by the confidence lack in various stages of the production process and the supply chain [3]. The Food and Agriculture Organization of the United Nations (FAO) report states that fisheries and aquaculture are recognized as one of the sectors most vulnerable to food fraud ${ }^{3}$ Adulteration in fish is committed when the fish is purposely placed on the market, for financial gain, to deceive the customer. There are different types of fraud that can occur at various points along the fish supply chain. The most common type involves intentional labeling and species substitution. In contrast, the ability to verify the authenticity and provenance of seafood, from catch to consumption, has become an essential requirement for sustainable, safe, and compatible commercial practices 4. Thus, quick and easy tools are needed to assess the authenticity of these products to address economic fraud throughout the supply chain.

The identification of fish species is a fundamental aspect to expose commercial fraud [5]. Previously, fish species were identified manually by observation and, therefore, it was necessary to remember or study various characteristics of fish to recognize them. Furthermore, the identification of fish species using the only appearance is difficult for humans, because species from the same family have a similar appearance, in addition to the environment and surrounding

\footnotetext{
1 https://ifs-productintegrity.com/wp-content/uploads/2020/09/IFS_Guideline_ Product_Fraud_Mitigation_V2_EN.pdf

${ }^{2}$ Available at https://secure.fera.defra.gov.uk/foodintegrity/index.cfm? sectionid=83

3 Available at http://www.fao.org/3/i8791en/I8791EN.pdf
} 
conditions, such as habitats, can change the fish color [6]. In this context, there is a need for techniques capable of performing a correct, automatic, and rapid identification of fish. When analyzing different species recognition systems to avoid counterfeiting, several decision criteria must be considered. These challenges include response time, accuracy, resolution, and features. According to Hridayami et. al, advanced technology, combined with artificial intelligence (AI) and deep learning methods, facilitates the task of recognizing fish species 7].

The Internet of Things (IoT) has presented itself as a novel network paradigm in a widespread and ubiquitous way, offering distributed and transparent services [8]. By IoT, many smart devices are connected, such as sensors, smartphones, and other smart devices. These devices can communicate with each other and exchange a large amount of information. However, in real-world scenarios, it is difficult to transmit large amounts of heterogeneous data, perceive complex environments from that data, and then make smart decisions in a timely manner 9. It is expected that AI technologies to be widely applied to IoT devices to collect, share, and analyze more data and valuable information [10]. AI, especially deep learning techniques, has become a proven success in several areas, including speech recognition, natural language processing, and computer vision. Advances in deep learning are a path to improve the perception capacity of IoT systems from a large number of heterogeneous data.

Deep learning is defined as a method of learning by representation, to preserve the data entry information, however, transforming it in a way that becomes useful for use. Thus, deep learning techniques refine the representation at various levels, using a deep artificial neural network and composed of multiple layers of neurons. Due to the efficient ability to learn resources, many complex problems can be solved quickly and effectively [11. Deep learning has several applications, such as automatic speech recognition, natural language processing, and image recognition [12. Convolutional neural networks (CNNs) are one of the most popular methods of deep learning [7. According to Sanghvi et al. this method remains the most sought after choice by computer scientists for image recognition, processing, and classification 13 .

CNNs are deep learning algorithms that receive an image as input, assign importance or weight to various aspects of it, and can differentiate one from the other. The name of this network type is due to the convolution operation. Convolution consists of extracting useful features from the input data, in this case, from the images. In addition to convolution, a CNN consists of the input layers, the grouping layer (pooling), the fully connected layer, and the output layer. CNNs are commonly used for image classification and recognition due to their high accuracy [13. Besides, this method is flexible and can adapt to new data received as the data set grows. Rachel and Varalakshmi claim that the main advantage of CNNs is that they automatically detect resources without any human intervention 12 .

In this context, this research proposes a fish recognition model for fraud prevention based on CNNs. Then, the dataset built in this study was submitted to CNN architectures for classification. Subsequently, tests and experiments 
were carried out to evaluate and validate the model with the best performance. Finally, a prototype of a web application is presented using a CNN model.

The main contributions of this paper are listed as follows:

- Create a data set with images of fish in real fishmonger environments.

- Develop CNN architectures using transfer learning and fine-tuning techniques.

- Compare and discuss the performance of CNNs in different scenarios.

- Propose a prototype web application to be developed on mobile and desktop devices with the CNN model with the best performance.

The rest of the paper is organized as follows. Section 2 addresses the relevant background in the topic and Section 3 elaborates on the most relevant related work for the study. The materials and methods are shown in Section 4 and results analysis and discussions are considered in Section 5 . Finally, Section 6 concludes the paper and presents suggestions for future works.

\section{Background}

This section summarizes the necessary concepts and background in the topic and purposes of this study.

\subsection{Artificial Intelligence}

During the past decades, AI has achieved great success with advances in cloud computing technologies, graphics processing unit (GPU) and other hardware enhancements [8. AI is a computer technology development advanced form. It fully simulates the operating process of the human brain and transforms technology to make it intellectualized [14. To some extent, AI applications can also effectively reduce people's workload and reduce human errors. In practice, this type of technology application cannot only carry out autonomous work according to the program, however, also perform creative work based on its characteristics.

Popular AI techniques include machine and deep learning methods, natural language processing, as well as expert systems. These different techniques are applied based on the characteristics of the data studied, to make the systems that use them intelligent [15].

\subsection{Deep Learning}

Deep learning has become a rapidly expanding research topic that was introduced to bring machine learning closer to artificial intelligence [16. However, deep learning runs through classic machine learning adding more complexity to the model. Also, it transforms data using various functions that allow the representation of data hierarchically, through various abstraction levels. 
Deep learning can be understood as the use of deep neural networks with several non-linear layers, in which resources are learned from the data through a general-purpose learning procedure. A strong advantage of approaches based on deep learning is resource learning, i.e., the automatic extraction of resources from raw data [17. Besides, it can solve more complex problems quickly, due to the more complex models used. Such models can increase the accuracy of the classification or reduce the error in the regression problems, as long as there are suitably large data sets describing the problem. These factors make it possible for deep learning structures to be flexible and adaptable to a wide variety of highly complex challenges. Although methods based on this approach have been used in many fields of research, the most effective field is the recognition of objects [18.

Among the various approaches to object recognition, deep learning stands out in image processing and data analysis, with promising results and great potential. One of the most important advantages of using deep learning in image processing is the reduced need for resource engineering since these are located through training and automatically. A disadvantage of this approach is the training time, which is generally longer. However, testing time is generally faster than other machine-based methods. Deep learning has several types of architecture, such as recurrent neural networks, recursive neural networks, and CNNs. This method is one of the most powerful deep learning tools for classifying images based on visual aids [19].

\subsection{Convolutional Neural Networks}

CNNs are a class of deep learning algorithms used to analyze data and one of the best learning algorithms for understanding image content. In addition, they showed exemplary performance in tasks related to image segmentation, classification, detection, and retrieval [20. Some of the areas of application for CNNs include object detection, video processing, image classification, and segmentation. Deep CNN's powerful learning ability is mainly due to the use of various stages of resource extraction that can automatically learn representations of the data. In addition, CNNs need less pre-processing time compared to other classification algorithms, since it can learn the characteristics automatically, whereas, in traditional methods, the process is done manually.

CNN consists of neurons that composed of weights, bias, and activation functions. Similar to other neural networks, CNN comprises input, hidden, and output layers, which perform operations that alter data to investigate specific resources. A basic CNN architecture has the input, convolution, and pooling layers, which alternate, fully connected, and output layers, as shown in Figure 1. CNN receives an image as input. The convolution layer acts as a resource extractor for the input images, whose dimensionality is then reduced by the grouping layers. The convolutional layers encode a number of lower-level features for more discriminating features, which transform one input image into another, highlighting specific patterns. The fully connected layer acts as a 
classifier, which exploits the high-level resources learned by CNN to classify input images into predefined classes. In short, this layer receives a vector as an input and produces another vector as an output.

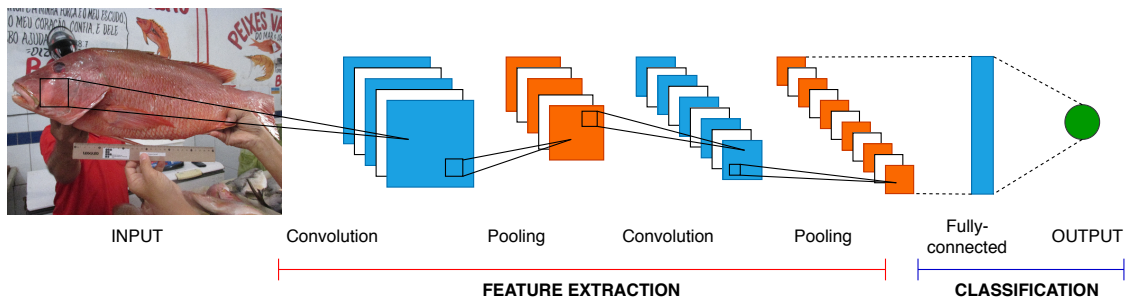

Fig. 1: Convolutional neural network architecture.

The availability of a large amount of data and the improvement of hardware technology has accelerated research on CNNs. Consequently, several new architectures have been proposed. The listed models of CNNs used in this study are covered in the next section. The criteria for choosing these models are based on the most recent state-of-the-art algorithms. Besides, these architectures are from public sources.

\subsubsection{VGG16}

Simonyan and Zisserman proposed the VGG architecture, which presented a simple and effective design principle for CNN architectures 21. The VGG16 has 23 layers to simulate the depth relationship with the representational capacity of the network. This CNN showed good results for both image classification and location problems, however, it is commonly recognized due to its simplicity, homogeneous topology and greater depth [20].

\subsubsection{Inception $V_{3}$}

The primary idea of InceptionV3 was to reduce the computational cost of deep networks without affecting the generalization. To that end, Szegedy et al. replaced large size filters with small, asymmetric filters and used the $1 \times 1$ convolution as a bottleneck before the large filters 22 . Such characteristics have become the main contributions of this architecture. This CNN is 159 layers deep.

\subsubsection{DenseNet121}

Elaborated by Huang et al., the DenseNet was proposed to solve the disappearance gradient problem 23. For this, this CNN used cross-layer connectivity, which connected each previous layer to the next arrival layer in a feed-forward 
way. Thus, feature maps for all previous layers were used as inputs for all subsequent layers. As DenseNet concatenates the characteristics of the previous layer instead of adding them, the network can gain the ability to explicitly differentiate between the information that is added to the network and that which is preserved 20. This architecture presents 121 layers of depth.

\subsubsection{MobileNet}

MobileNet was designed by Howard et al. for use on mobile devices. For this, this CNN uses in-depth separable convolutions [24]. This fact significantly reduces the number of parameters when compared to networks with regular convolutions of the same depth. This results in light deep neural networks. By defining the network in such simple terms, it is possible to easily explore its structure and outline a good network [25].

\subsubsection{Xception}

Xception was proposed by Chollet and it can be considered an architecture of extreme beginning, which explores the idea of separable convolution in-depth [26]. This architecture modified the original initial block making it wider and replacing the different spatial dimensions with a single dimension, followed by a $1 \times 1$ convolution to regulate computational complexity. Xception is 126 layers deep. Although the transformation strategy adopted by this CNN does not reduce the number of parameters, it makes learning more efficient and results in better performance [20].

\section{Related Works}

Several approaches using CNNs have been proposed for the recognition of fish species, achieving promising results. In this section, some studies related to the theme of this research are presented.

Santos and Gonçalves presented a CNN-based method for the recognition of fish species in the Brazilian Pantanal 27. A CNN, composed of three branches that classify fish by family and order, is proposed to improve the recognition of species with similar characteristics. The results in the data set showed that the proposed method obtained an accuracy of 0.873 and 0.864 using a traditional CNN. The authors recognized 68 species of fish. Despite presenting a variability of images in the dataset, encouraging CNN to adapt to different data inputs, the focus on extracting images in real environments has been shifted. Thus, the model can obtain inferior performance when receiving low-quality images, in environments different from the images presented, among other situations.

Masuda et al. proposed an advanced method of identifying fish species using images of fish and their meristic characteristics. The authors developed a model of multiple entries that presents images and, also, data of characteristics, aiming to become an advanced system of identification of fish species. To show 
the accuracy of the proposed model, the study compared with models that used only images or just meristic characters. As a result, it was clarified that the use of two or more types of meristic characters provided greater precision than the use of images only $[\underline{6}$. The authors worked with the identification of nine species of fish. However, as exposed in the study, the number of images by species is unbalanced. Then, some species have about double or triple the images of other species, causing overfitting. Therefore, the algorithm erroneously classifies species of fish for presenting a few training data.

The research of Rauf et al. showed a deep learning structure based on the CNN method for identifying fish species [19. The proposed CNN architecture contains 32 deep layers to extract valuable and discriminating features from the image. To test the model performance, the authors created a data set called Fish-Pak, containing 915 images. To validate the proposed CNN, the authors compared their results with other structures of deep learning, involving VGG16, LeNet5, AlexNet, GoogleNet, and ResNet50. Empirical analyzes revealed that the proposed method achieves satisfactory performance and exceeds existing methods. In the dataset of this research, six species of fish were considered, segmenting the images in specific regions that characterize each species studied. However, the dataset is also unbalanced. This characteristic can result in an inflexible algorithm, making it ineffective for certain species of fish.

Vo et al. proposed the use of a CNN in conjunction with image processing techniques to automatically classify lobsters, aiming to control fraud during the marketing chain [4]. By using a $\mathrm{CNN}$, the research aims to enhance previous research on the classification of lobsters by establishing a reliable and flexible method of traceability to address different supply chain contexts. Although the focus of the research is the recognition of lobsters, the research generates important contributions. In the proposed approach, a pre-trained Mask-RCNN model was adopted to extract regions of interest from the lobster images. To prove the effectiveness, the proposed method was validated against a set of image data collected on a lobster processor and tested in the mobile application environment. As a challenge, it is evident that the dataset is reduced, with the training set consisting of 150 images and the validation set consisting of 90 images. Also, the capture of the images was carried out by a set of three cameras placed from top to bottom and the appropriate distance was also defined to take the photo and, thus, the algorithm would be able to recognize the lobster.

Rossi et al. presented the FishAPP, a cloud-based infrastructure for recognizing fish species [5]. The approach is through a mobile application developed for Android and iOS, allowing the user to take photos of an entire fish and send them for remote analysis. A cloud system performs image processing and is then subjected to a CNN capable of analyzing the images obtained and performing classification on fish species. The preliminary results obtained from the available data set provided encouraging results. The study imposes, on the user, a series of guidelines when taking the photo of the fish, such as the photo must include the complete fish, it must be photographed from the side and the tail fin must be placed anatomically and relaxed. Such factors restrict the 
relevance of the application, as it does not suit different and possible real-life scenarios.

From the studies presented, there are still challenges for the task of recognizing fish. They are $i$ ) dataset with limited number of images; ii) imbalance in the quantity of images by species, and iii) restriction of images in real scenarios and under restricted conditions. Therefore, the objective of this study is to propose a model to combat fish fraud using CNNs, thus contributing to overcoming the challenges listed.

\section{Materials and Methods}

In this section, the materials and methods necessary for the implementation of this research proposal are presented.

Figure 2 shows the steps taken to achieve the objective of this research. These phases comprise five main activities, namely, elaboration of the dataset (1), implementation of the CNN architectures (2), training and testing of the architectures (3), evaluation of the results (4), and the web application mockup (5). These steps are explained in detail below.

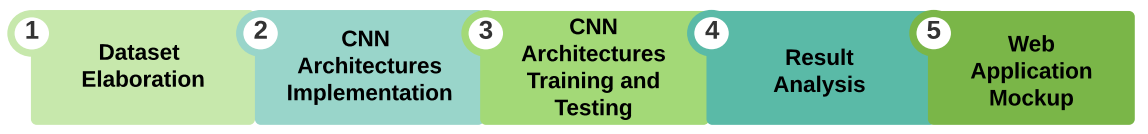

Fig. 2: Flowchart of research steps.

Step 1 comprises the elaboration of the dataset. For this, a data set with 338 images was built, divided into 8 species of marine fish, commonly found on the East Coast of Ceará, Brazil. The images were captured between August 2019 and August 2020 using the Canon camera, Smartphones iPhone 7 Plus, and Xiaomi RedMi Note 8 devices. Besides, the photos were collected at fish sales establishments in the city of Aracati, CE, Brazil. Figure 3 shows some examples of images in the data set. These images generally include elements other than fish, such as objects, the human body, among others. These variations encourage the model to select identification characteristics that do not depend on the orientation or focus of the image being classified.

After the elaboration of the dataset, the CNN architectures were implemented, which is step 2 of the proposal. For the coding of the models, the TensorFlow 4 defined as an open-source platform for machine learning, was used. Also, the Keras 5 was also used, which is a high-level API of the TensorFlow, used to create and train deep learning models. Concerning the chosen CNNs, this study opted for the following architectures, namely, VGG16, InceptionV3,

\footnotetext{
4 Available at https://www.tensorflow.org.

5 Available at https://keras.io/
} 

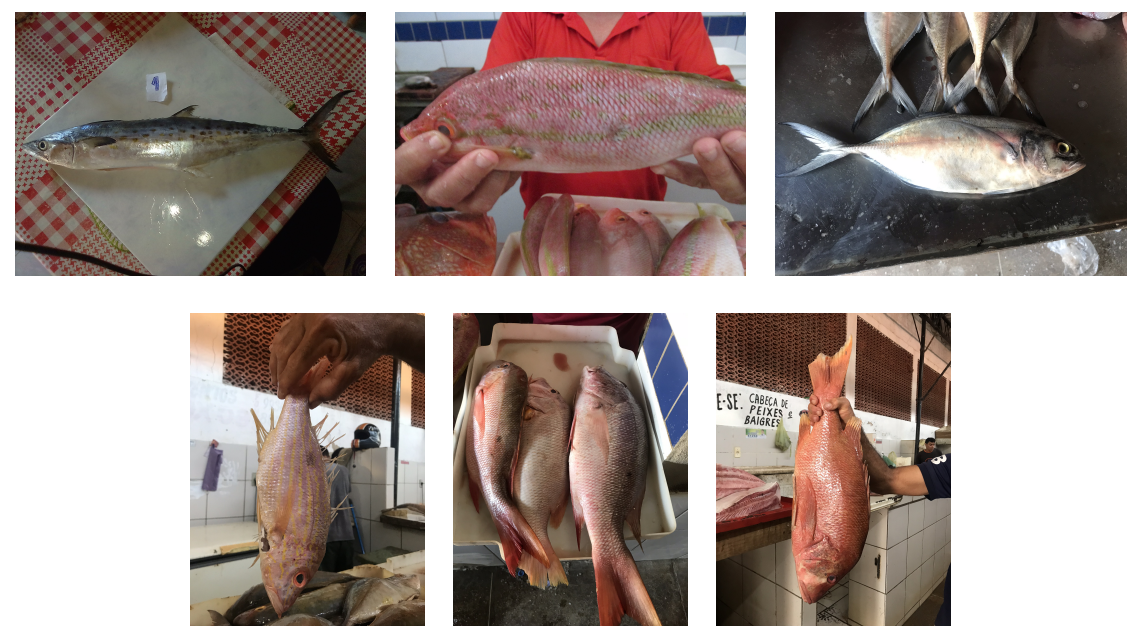

Fig. 3: Sample images from the dataset.

DenseNet121, MobileNet, and Xception. These CNNs were chosen because they are the most recent state-of-the-art networks in the area. Besides, these algorithms are available at the API Keras. The architectures were implemented using two techniques, namely, transfer learning and fine-tuning. Transfer learning comprises the use of a pre-trained model applied to a new problem. In summary, a pre-trained neural network is used for a new application. The fine-tuning, on the other hand, reuse the weights of the original network in the new model and, using a new data set, a new training step is carried out to adapt the weights to the new dataset. In this research, the initial layers of pre-trained CNNs served as a feature extractor. Then, the top of the network was modified to suit the classes of the model and new training was carried out.

Step 3 relates to the task of training and testing the models. Figure 4 shows the distribution of images after the elaboration of the dataset. It can be seen that this is partially balanced, presenting an average of 42 images per class. The data set was divided using the ratio of $70 \%$ for training and $30 \%$ for testing to training the architectures, respectively. Table 1 shows the division of fish species by family and scientific name. In summary, this study analyzed 4 types of families distributed in 8 types of fish. It is important to highlight that species from the same family have very similar characteristics and the model has the challenge of finding the attributes that differentiate each species.

With the trained and tested CNN models, step 4 was reached, the analysis of results. Further details of the results are shown in Section 5. Finally, in step 5, the web application mockup was prepared from the software Adobe XD for building prototypes. The mockup presents the layout of a software graphical interface. It is useful to demonstrate the use and the final result of an application. In this study, the web application prototype was built, aiming 


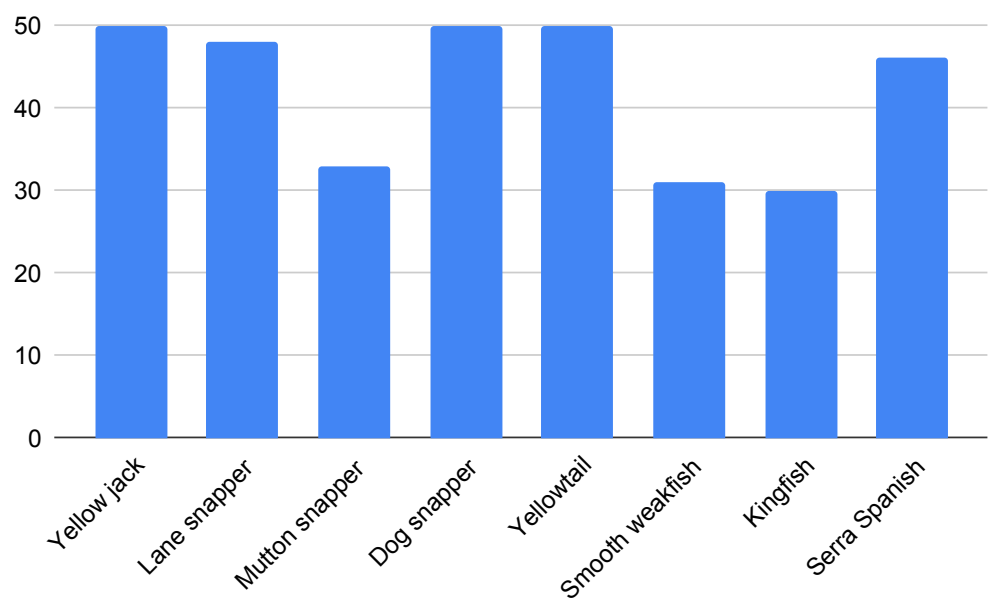

Fig. 4: Distribution of images in the dataset.

\begin{tabular}{|l|l|l|}
\hline Family & Specie (Popular Name) & Scientific Name \\
\hline Carangidae & Yellow jack & Carangoides bartholomaei \\
\hline \multirow{4}{*}{ Lutjanidae } & Lane snapper & Lutjanus synagris \\
\cline { 2 - 3 } & Mutton snapper & Lutjanus analis \\
\cline { 2 - 3 } & Dog snapper & Lutjanus jocu \\
\cline { 2 - 3 } & Yellowtail snapper & Lutjanus chrysurus \\
\hline \multirow{2}{*}{ Sciaenidae } & Smooth weakfish & Cynoscion leiarchus \\
\hline \multirow{2}{*}{ Scombridae } & Kingfish & Scomberomorus cavalla \\
\cline { 2 - 3 } & Serra Spanish mackerel & Scomberomorus brasiliensis \\
\hline
\end{tabular}

Table 1: Species distribution by family.

at future implementation based on the CNN model with the best performance obtained in this research.

\section{Discussion and Result Analysis}

Next, the results obtained from the CNN test stage are shown.

Two scenarios were elaborated to obtain the results shown in this section. The first, in which the test dataset was submitted during the training stages at the top of the network; and the second, in which the test data set was submitted only in the test stage of the model. The results achieved for each scenario are presented below.

Considering scenario 1, Figure 5 shows the confusion matrices of the CNNs. The confusion matrix is a table that allows you to view the frequency of errors and hits in the model and is useful for analyzing its performance. Considering 
Figure 5a, which shows the matrix of the VGG16 network, it is observed that there was a good performance for the species Lane snapper (13), Yellowtail snapper (13), Dog snapper (14), and Yellow jack (17). For the species Mutton snapper (4), Kingfish (5), and Serra Spanish mackerel (5) there was a low prediction. Figure $5 \mathrm{~b}$ shows the result for InceptionV3, with the species Serra Spanish mackerel (11), Yellow jack (12), Lane snapper (13), Dog snapper (13), and Yellowtail snapper (13) the greatest predictions. Kingfish (7) and Smooth weakfish (7), in turn, had the lowest predictions. The DenseNet121 matrix is shown in Figure 5c. In this Figure, Lane snapper (13), Yellow jack (14), Dog snapper (16), and Yellowtail snapper (16) were the most correctly predicted species, while Kingfish (7) and Mutton snapper (7) again underperformed. Figure 5d shows the result for MobileNet. The species Yellow jack (16), Serra Spanish mackerel (17), and Yellowtail snapper (21) reached a higher frequency of correctly predicted values. In this $\mathrm{CNN}$, in particular, in addition to the species Mutton snapper (6) and Kingfish (7) showing inferior performance, Dog snapper (9) also obtained the same behavior. Finally, Figure 5e shows the results for Xception. In this matrix, Yellow jack (15), Yellowtail snapper (18), and Dog snapper (20) obtained the highest frequencies of correctly predicted values. In contrast, Mutton snapper (5) and Kingfish (7) had low correct predictions. Briefly, CNNs VGG16, InceptionV3, and DenseNet121 showed better results considering the confusion matrices. Concomitantly, the species with the largest number of images in the dataset performed better, namely, Lane snapper, Dog snapper, Yellowtail snapper, and Yellow jack. On the other hand, the species Kingfish and Mutton snapper showed inferior performance, a fact that may be related to the quality of the images.

For the scenario 1, Table 2 shows the metrics precision, recall, and F-Score. Table $2 \mathrm{a}$ presents the results for the precision metric. Accuracy indicates the relationship between positive predictions correctly and all positive predictions (including false ones). In general, the species that obtained the best performance independently of CNN were Dog snapper, Yellowtail snapper, Yellow jack, and Smooth weakfish. Regarding the architectures, the Xception obtained better precision with an average of 0.89 . Table $2 \mathrm{~b}$ shows performance for the recall metric. This parameter lists the positive forecasts correctly made and all forecasts, i.e., true positives and false negatives. In this table, rates fluctuated for all classes and architectures. The CNN with the best recall was MobileNet, with an average of 0.85 . The F-Score metric is shown in Table 2c This standard, in turn, is the harmonic mean between precision and recall, which indicates the overall quality of the model. Again, rates varied for classes and CNNs, with the Xception architecture performing better, averaging 0.85. In light of the above, the importance of the number of images per class is perceived to assist in the training of the model and, consequently, improve its performance when subjected to tests.

Another result obtained was the accuracy of the CNN architectures. Accuracy represents how close the prediction is to the correct result. Thus, the greater the accuracy, the closer to the real value is the result found. Table 3 


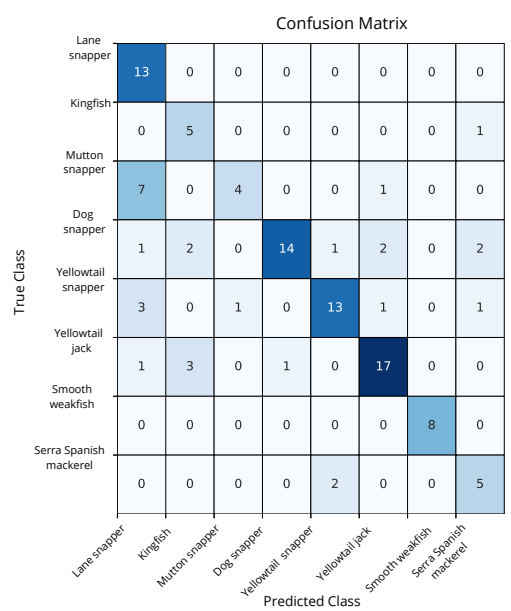

(a) VGG16

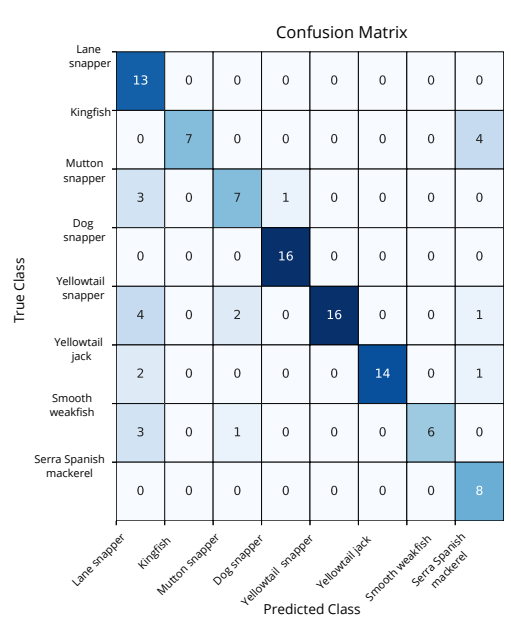

(c) DenseNet121

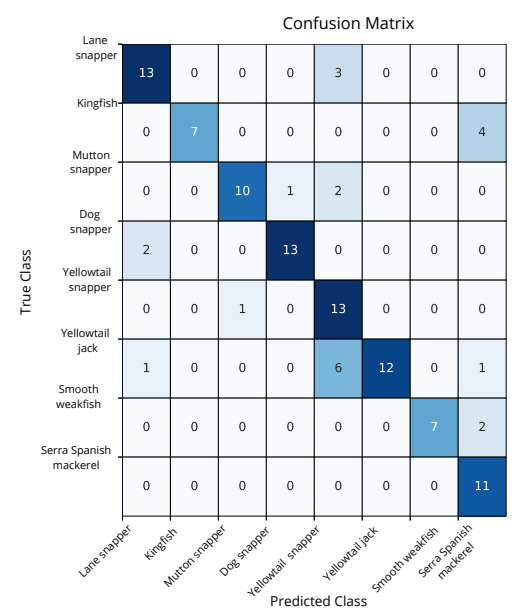

(b) InceptionV3

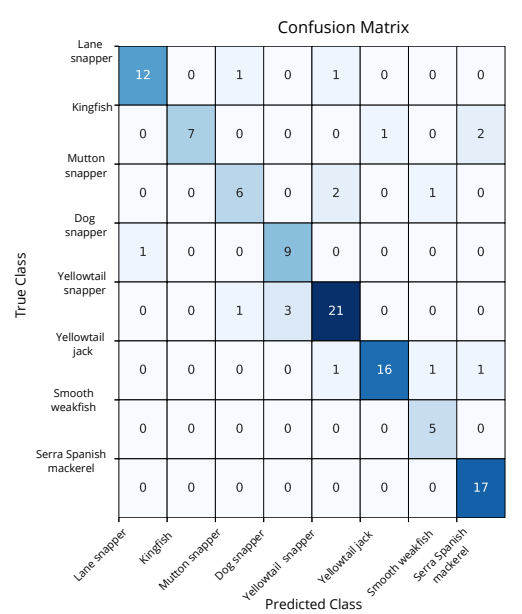

(d) MobileNet

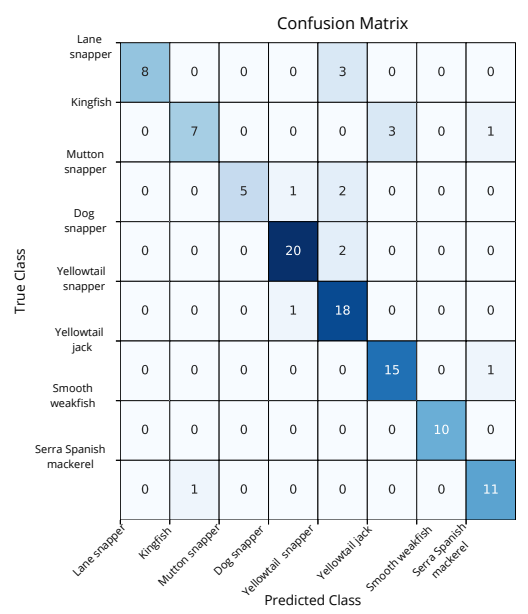

(e) Xception

Fig. 5: Confusion matrices for scenario 1. 


\begin{tabular}{|l|c|c|c|c|c|c|c|c|}
\cline { 2 - 9 } \multicolumn{1}{c|}{} & $\begin{array}{l}\text { Lane } \\
\text { snapper }\end{array}$ & Kingfish & $\begin{array}{l}\text { Mutton } \\
\text { snapper }\end{array}$ & $\begin{array}{l}\text { Dog } \\
\text { snapper }\end{array}$ & $\begin{array}{l}\text { Yellowtail } \\
\text { snapper }\end{array}$ & $\begin{array}{l}\text { Yellow } \\
\text { jack }\end{array}$ & $\begin{array}{l}\text { Smooth } \\
\text { weakfish }\end{array}$ & $\begin{array}{l}\text { Serra Spanish } \\
\text { mackerel }\end{array}$ \\
\hline VGG16 & 0.52 & 0.50 & 0.80 & 0.93 & 0.81 & 0.81 & 1.00 & 0.56 \\
\hline InceptionV3 & 0.81 & 1.00 & 0.91 & 0.93 & 0.54 & 1.00 & 1.00 & 0.61 \\
\hline DenseNet121 & 0.52 & 1.00 & 0.70 & 0.94 & 1.00 & 1.00 & 1.00 & 0.57 \\
\hline MobileNet & 0.92 & 1.00 & 0.75 & 0.75 & 0.84 & 0.94 & 0.71 & 0.85 \\
\hline Xception & $\mathbf{1 . 0 0}$ & $\mathbf{0 . 8 8}$ & $\mathbf{1 . 0 0}$ & $\mathbf{0 . 9 1}$ & $\mathbf{0 . 7 2}$ & $\mathbf{0 . 8 3}$ & $\mathbf{1 . 0 0}$ & $\mathbf{0 . 8 5}$ \\
\hline
\end{tabular}

(a) Precision Metric.

\begin{tabular}{|l|c|c|c|c|c|c|c|c|}
\cline { 2 - 8 } \multicolumn{1}{c|}{} & $\begin{array}{l}\text { Lane } \\
\text { snapper }\end{array}$ & Kingfish & $\begin{array}{l}\text { Mutton } \\
\text { snapper }\end{array}$ & $\begin{array}{l}\text { Dog } \\
\text { snapper }\end{array}$ & $\begin{array}{l}\text { Yellowtail } \\
\text { snapper }\end{array}$ & $\begin{array}{l}\text { Yellow } \\
\text { jack }\end{array}$ & $\begin{array}{l}\text { Smooth } \\
\text { weakfish }\end{array}$ & $\begin{array}{l}\text { Serra Spanish } \\
\text { mackerel }\end{array}$ \\
\hline VGG16 & 1.00 & 0.83 & 0.33 & 0.64 & 0.68 & 0.77 & 1.00 & 0.71 \\
\hline InceptionV3 & 0.81 & 0.64 & 0.77 & 0.87 & 0.93 & 0.60 & 0.78 & 1.00 \\
\hline DenseNet121 & 1.00 & 0.64 & 0.64 & 1.00 & 0.70 & 0.82 & 0.60 & 1.00 \\
\hline MobileNet & $\mathbf{0 . 8 6}$ & $\mathbf{0 . 7 0}$ & $\mathbf{0 . 6 7}$ & $\mathbf{0 . 9 0}$ & $\mathbf{0 . 8 4}$ & $\mathbf{0 . 8 4}$ & $\mathbf{1 . 0 0}$ & $\mathbf{1 . 0 0}$ \\
\hline Xception & 0.73 & 0.64 & 0.62 & 0.91 & 0.95 & 0.94 & 1.00 & 0.92 \\
\hline
\end{tabular}

(b) Recall Metric.

\begin{tabular}{|l|c|c|c|c|c|c|c|c|}
\cline { 2 - 8 } \multicolumn{1}{c|}{} & $\begin{array}{l}\text { Lane } \\
\text { snapper }\end{array}$ & Kingfish & $\begin{array}{l}\text { Mutton } \\
\text { snapper }\end{array}$ & $\begin{array}{l}\text { Dog } \\
\text { snapper }\end{array}$ & $\begin{array}{l}\text { Yellowtail } \\
\text { snapper }\end{array}$ & $\begin{array}{l}\text { Yellow } \\
\text { jack }\end{array}$ & $\begin{array}{l}\text { Smooth } \\
\text { weakfish }\end{array}$ & $\begin{array}{l}\text { Serra Spanish } \\
\text { mackerel }\end{array}$ \\
\hline VGG16 & 0.68 & 0.62 & 0.47 & 0.76 & 0.74 & 0.79 & 1.00 & 0.63 \\
\hline InceptionV3 & 0.81 & 0.78 & 0.83 & 0.90 & 0.68 & 0.75 & 0.88 & 0.76 \\
\hline DenseNet121 & 0.68 & 0.78 & 0.67 & 0.97 & 0.82 & 0.90 & 0.75 & 0.73 \\
\hline MobileNet & 0.89 & 0.82 & 0.71 & 0.82 & 0.84 & 0.89 & 0.83 & 0.92 \\
\hline Xception & $\mathbf{0 . 8 4}$ & $\mathbf{0 . 7 4}$ & $\mathbf{0 . 7 7}$ & $\mathbf{0 . 9 1}$ & $\mathbf{0 . 8 2}$ & $\mathbf{0 . 8 8}$ & $\mathbf{1 . 0 0}$ & $\mathbf{0 . 8 8}$ \\
\hline
\end{tabular}

(c) F-Score Metric

Table 2: Metrics for scenario 1.

shows that Xception presented better accuracy, with a rate of $86 \%$, as well as better results for the metrics precision and F-Score.

\begin{tabular}{|l|c|}
\cline { 2 - 2 } \multicolumn{1}{c|}{} & Accuracy \\
\hline VGG16 & 0.72 \\
\hline InceptionV3 & 0.78 \\
\hline DenseNet121 & 0.79 \\
\hline MobileNet & 0.85 \\
\hline Xception & $\mathbf{0 . 8 6}$ \\
\hline
\end{tabular}

Table 3: Accuracy metric for scenario 1.

The results for scenario 2 will now be presented. The first of these is the confusion matrices, shown in Figure 6. When considering the VGG16 architecture, the species Lane snapper (13), Serra Spanish mackerel (14), Dog snapper (16), and Yellow jack (18) have the highest frequencies of correct predictions, as shown in Figure 6a. Kingfish (4) and Mutton snapper (6), however, obtained the lowest frequencies of correct values. Figure 6b shows the confusion matrix of InceptionV3. In this Figure, Serra Spanish mackerel (10), Lane snapper (11), Dog snapper (11), Yellowtail snapper (12), and Yellow jack (13) had better results, while Mutton snapper (6) again presented an irregular behavior. The confusion matrix of DenseNet121 is shown in Figure 5c For this CNN, Dog snapper (15), Yellowtail snapper (17), and Yellow jack (23) obtained more correct predictions. However, Lane snapper (6), Kingfish (6), and Mutton snapper (6) underperformed. Figure 6d shows the results for the MobileNet 
architecture. In this result, the classes Lane snapper (13), Yellow jack (14), Yellowtail snapper (15) and Dog snapper (17) have more positive predictions, while Kingfish (8), Mutton snapper (8), and Smooth weakfish (8) resulted in fewer values correct. The matrix for CNN Xcpetion is shown in Figure 6e which shows that Lane snapper (12), Dog snapper (13), Yellowtail snapper (13), Yellow jack (16), and Serra Spanish mackerel (16) obtained positive predictions more frequently. However, Mutton snapper (5) and Kingfish (6) showed low performance. Given the above, it appears that the Xception and MobileNet CNNs showed better performances in terms of the confusion matrix. Once more, it is important to note that species with a greater number of images tend to have better results.

Table 4 presents the metrics precision, recall, and F-Score for scenario 2. Considering the precision metric, the Dog snapper species and Yellowtail snapper obtained higher rates, according to Table 4a. The CNN DenseNet121 performed better among classes, averaging 0.87 . When observing the recall metric in Table $4 \mathrm{~b}$ it appears that all species had varying rates for all CNNs. Once more, DenseNet121 achieved better efficiency with an average rate of 0.84. The metric F-Score, shown in Table 4c, shows that the classes Yellow jack and Smooth weakfish obtained the best results. DenseNet121 also had the best result, averaging 0.83 . In short, the DenseNet121 architecture performed better for the metrics in Table 4

\begin{tabular}{|l|c|c|c|c|c|c|c|c|}
\cline { 2 - 9 } \multicolumn{1}{c|}{} & $\begin{array}{l}\text { Lane } \\
\text { snapper }\end{array}$ & Kingfish & $\begin{array}{l}\text { Mutton } \\
\text { snapper }\end{array}$ & $\begin{array}{l}\text { Dog } \\
\text { snapper }\end{array}$ & $\begin{array}{l}\text { Yellowtail } \\
\text { snapper }\end{array}$ & $\begin{array}{l}\text { Yellow } \\
\text { jack }\end{array}$ & $\begin{array}{l}\text { Smooth } \\
\text { weakfish }\end{array}$ & $\begin{array}{l}\text { Serra Spanish } \\
\text { mackerel }\end{array}$ \\
\hline VGG16 & 0.81 & 1.00 & 0.60 & 0.89 & 0.50 & 1.00 & 0.78 & 0.88 \\
\hline InceptionV3 & 0.73 & 0.50 & 0.46 & 0.92 & 0.80 & 0.93 & 0.90 & 0.71 \\
\hline DenseNet121 & $\mathbf{1 . 0 0}$ & $\mathbf{0 . 6 7}$ & $\mathbf{0 . 8 6}$ & $\mathbf{0 . 8 3}$ & $\mathbf{0 . 7 1}$ & $\mathbf{0 . 9 2}$ & $\mathbf{1 . 0 0}$ & $\mathbf{1 . 0 0}$ \\
\hline MobileNet & 0.81 & 0.62 & 0.73 & 1.00 & 0.79 & 0.82 & 1.00 & 0.88 \\
\hline Xception & 1.00 & 1.00 & 0.33 & 1.00 & 0.76 & 1.00 & 1.00 & 0.70 \\
\hline
\end{tabular}

(a) Precision Metric

\begin{tabular}{|l|c|c|c|c|c|c|c|c|}
\cline { 2 - 9 } \multicolumn{1}{c|}{} & $\begin{array}{l}\text { Lane } \\
\text { snapper }\end{array}$ & Kingfish & $\begin{array}{l}\text { Mutton } \\
\text { snapper }\end{array}$ & $\begin{array}{l}\text { Dog } \\
\text { snapper }\end{array}$ & $\begin{array}{l}\text { Yellowtail } \\
\text { snapper }\end{array}$ & $\begin{array}{l}\text { Yellow } \\
\text { jack }\end{array}$ & $\begin{array}{l}\text { Smooth } \\
\text { weakfish }\end{array}$ & $\begin{array}{l}\text { Serra Spanish } \\
\text { mackerel }\end{array}$ \\
\hline VGG16 & 0.76 & 0.57 & 0.75 & 0.70 & 0.82 & 0.90 & 1.00 & 0.88 \\
\hline InceptionV3 & 0.85 & 0.80 & 0.86 & 0.65 & 0.67 & 0.76 & 0.82 & 0.62 \\
\hline DenseNet121 & $\mathbf{0 . 5 5}$ & $\mathbf{0 . 8 6}$ & $\mathbf{0 . 7 5}$ & $\mathbf{0 . 8 8}$ & $\mathbf{0 . 9 4}$ & $\mathbf{0 . 8 8}$ & $\mathbf{1 . 0 0}$ & $\mathbf{0 . 8 6}$ \\
\hline MobileNet & 0.93 & 1.00 & 0.80 & 0.81 & 0.94 & 0.82 & 1.00 & 0.47 \\
\hline Xception & 0.80 & 0.46 & 1.00 & 0.72 & 0.68 & 1.00 & 1.00 & 1.00 \\
\hline
\end{tabular}

(b) Recall Metric.

\begin{tabular}{|l|c|c|c|c|c|c|c|c|}
\cline { 2 - 8 } \multicolumn{1}{c|}{} & $\begin{array}{l}\text { Lane } \\
\text { snapper }\end{array}$ & Kingfish & $\begin{array}{l}\text { Mutton } \\
\text { snapper }\end{array}$ & $\begin{array}{l}\text { Dog } \\
\text { snapper }\end{array}$ & $\begin{array}{l}\text { Yellowtail } \\
\text { snapper }\end{array}$ & $\begin{array}{l}\text { Yellow } \\
\text { jack }\end{array}$ & $\begin{array}{l}\text { Smooth } \\
\text { weakfish }\end{array}$ & $\begin{array}{l}\text { Serra Spanish } \\
\text { mackerel }\end{array}$ \\
\hline VGG16 & 0.79 & 0.73 & 0.67 & 0.78 & 0.62 & 0.95 & 0.88 & 0.88 \\
\hline InceptionV3 & 0.79 & 0.62 & 0.60 & 0.76 & 0.73 & 0.84 & 0.86 & 0.67 \\
\hline DenseNet121 & $\mathbf{0 . 7 1}$ & $\mathbf{0 . 7 5}$ & $\mathbf{0 . 8 0}$ & $\mathbf{0 . 8 6}$ & $\mathbf{0 . 8 1}$ & $\mathbf{0 . 9 0}$ & $\mathbf{1 . 0 0}$ & $\mathbf{0 . 9 2}$ \\
\hline MobileNet & 0.87 & 0.76 & 0.76 & 0.89 & 0.86 & 0.82 & 1.00 & 0.61 \\
\hline Xception & 0.89 & 0.63 & 0.50 & 0.84 & 0.72 & 1.00 & 1.00 & 0.82 \\
\hline
\end{tabular}

(c) F-Score Metric.

Table 4: Metrics for scenario 2.

The final accuracy of the CNN models is shown in Table 5 . It is observed that for scenario 2, the DenseNet121 architecture presented better accuracy, with 


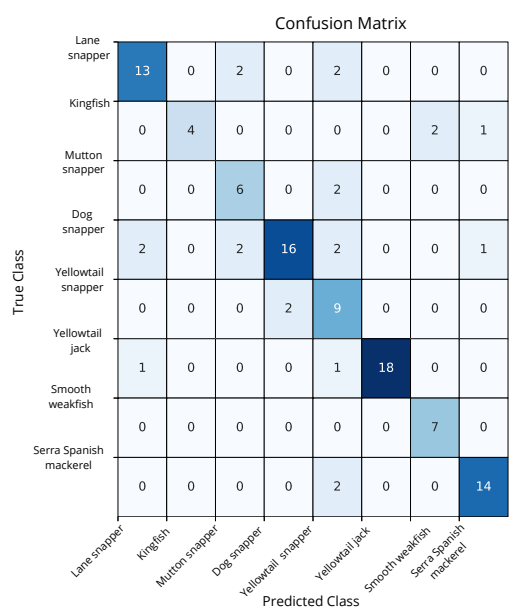

(a) VGG16

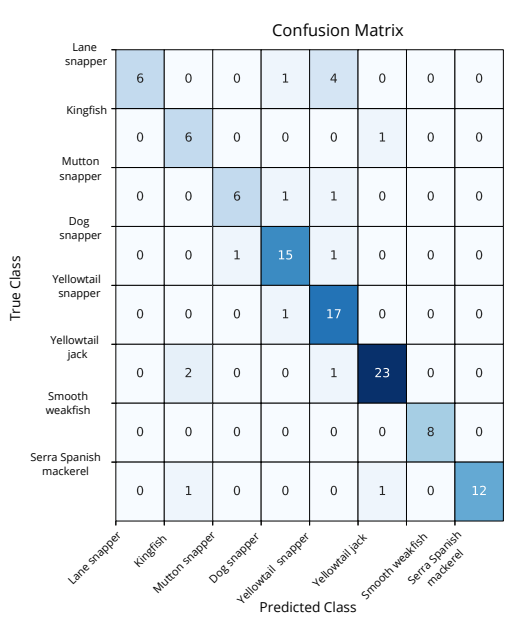

(c) DenseNet121

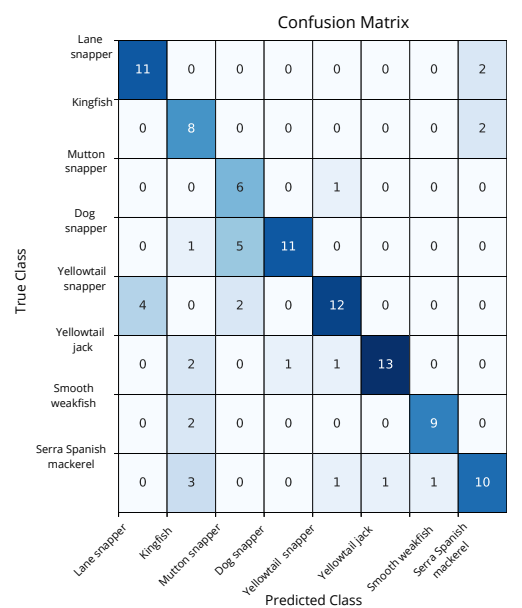

(b) InceptionV3

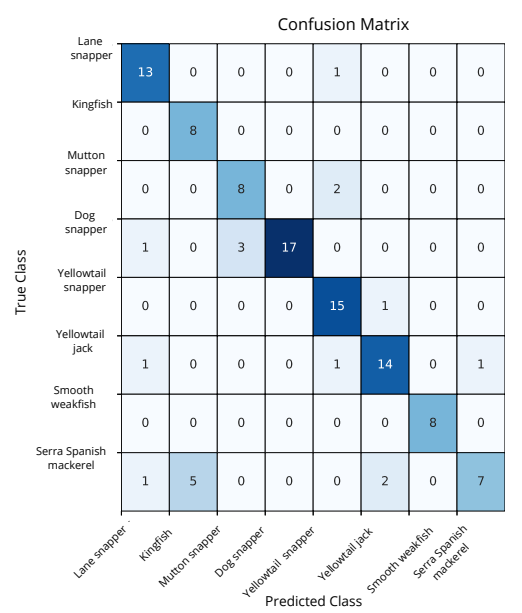

(d) MobileNet

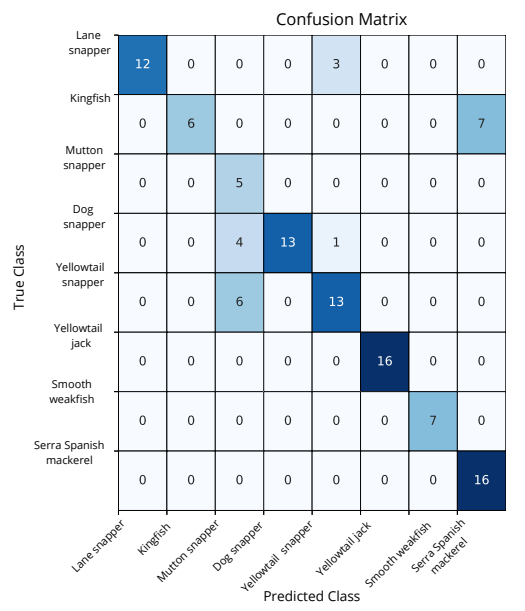

(e) Xception

Fig. 6: Confusion matrices for scenario 2. 
a rate of $85 \%$, also demonstrating its effectiveness for the metrics previously analyzed.

\begin{tabular}{|l|c|}
\cline { 2 - 2 } \multicolumn{1}{c|}{} & Accuracy \\
\hline VGG16 & 0.79 \\
\hline InceptionV3 & 0.73 \\
\hline DenseNet121 & $\mathbf{0 . 8 5}$ \\
\hline MobileNet & 0.82 \\
\hline Xception & 0.80 \\
\hline
\end{tabular}

Table 5: Accuracy metric for scenario 2.

In summary, it can be seen that, in scenario 1, the CNN Xception performed better with $86 \%$ accuracy, and in scenario 2, the CNN DenseNet121 achieved $85 \%$ performance in terms of accuracy. The difference, in terms of accuracy for both scenarios, is minimal, although for each scenario there was a different CNN.

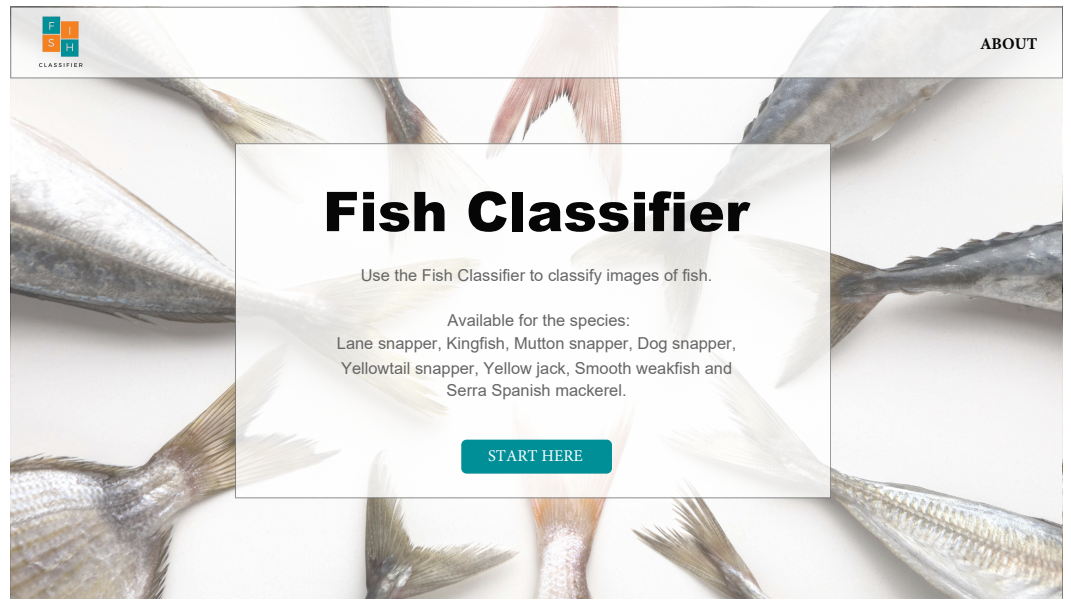

Fig. 7: Home screen of the web application.

After obtaining the CNN model with the best result from the tests performed, the mockup for the web application was developed, which is detailed as follows. On the home screen, shown in Figure 7, the user is introduced to the Fish Classifier system. It was decided to establish a pattern of screens, starting from the initial screen. Relevant detail is that the system logo takes you to the home page, anywhere in the application. The "About" button, in turn, redirects you to the screen with information from the Fish Classifier. Also on the home screen, there is a brief text about the application and the species available for classification. The user can perform the classification in the button "Start Here!". The next screen is the classification screen, shown 


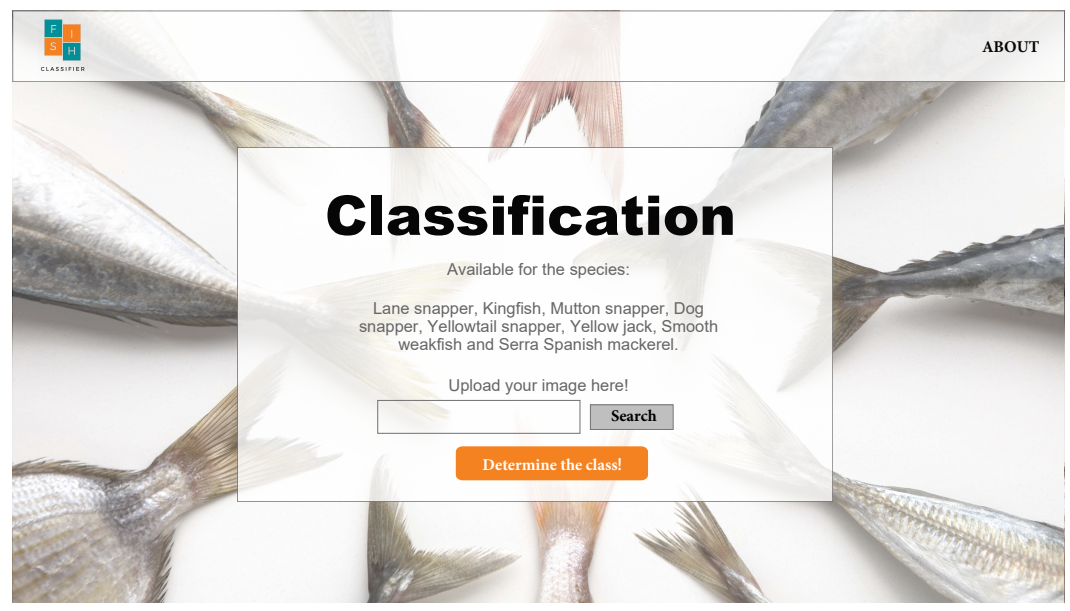

Fig. 8: Classification screen of the web application.

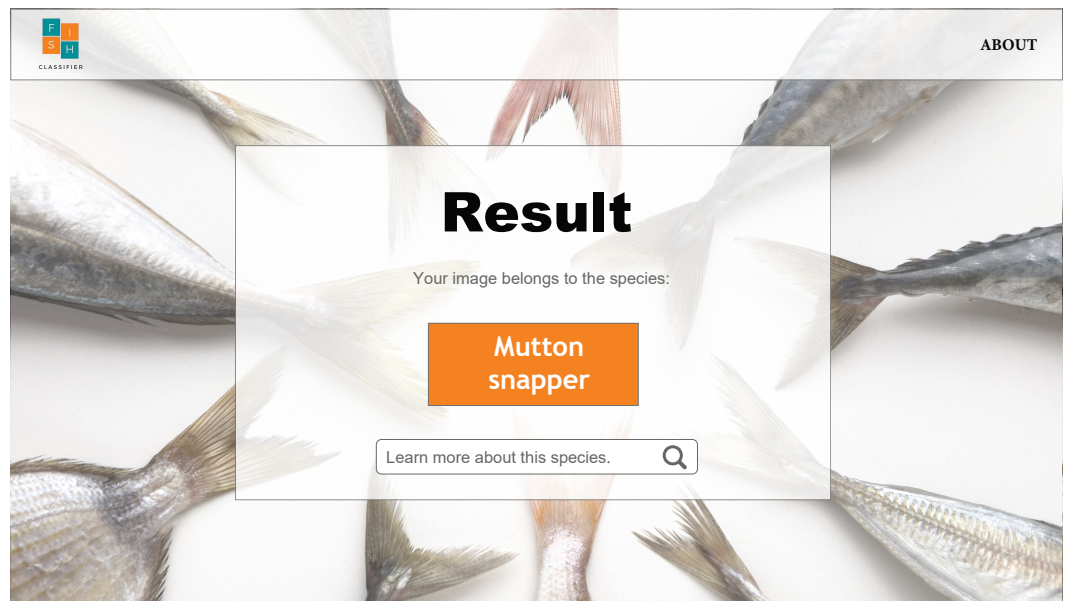

Fig. 9: Result screen of the web application.

in Figure 8, On this screen, the user can upload the fish image that he wants to classify. Then, the user must click on the "Determine the Class!" Button. Then, the system redirects to the result page, shown in Figure 9. The user obtains the result of the classifier, indicating the species to which the image belongs. If he wants to know more about the species, just click on the option "Learn more about this species". Finally, on the About screen, shown in Figure 10. a text about the application is presented with general information, such as description, objective, target audience, species available for classification, among others. The user can return to the system home screen by clicking on the Fish Classifier logo. When building the mockup, the objective was to show a prototype of the system to be implemented with the model that presented 


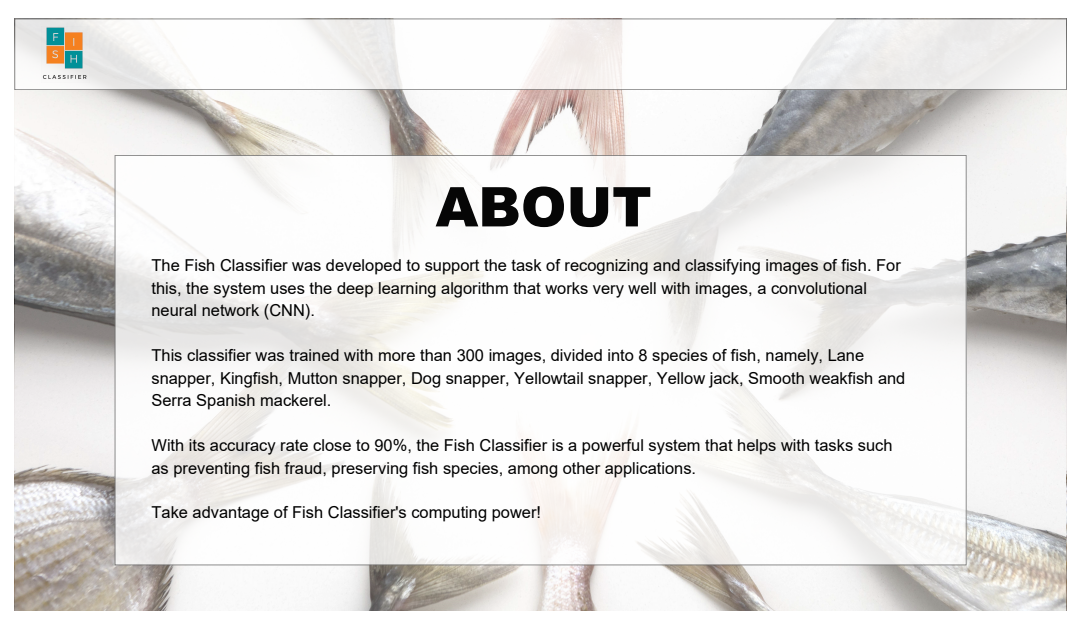

Fig. 10: About screen of the web application.

the best performance, in this case, Xception with an accuracy of $86 \%$. When developing the web application, it is expected that it has a simple and, at the same time, intuitive interface for the user, according to the prototype shown in this section.

\section{Conclusion and Future Works}

This paper presented a fish recognition model for preventing fish fraud. From the above, it was possible to build a dataset with images in real environments. Furthermore, after the implementation and comparison of the results of the CNNs, it was found that the Xception architecture achieved better performance with $86 \%$ accuracy. It was also possible to build a web application mockup. Given the above, it is concluded that the model can be applied to prevent fraud in the fish supply chain.

The study limitations are related to the construction of the data set because collecting images of certain species is very time-consuming. Another challenge was to understand the performance of each architecture and its implementation, from the choice stage to the analysis of the results.

Suggestions for further works are based on increasing the accuracy rate of the model and on the coding of the web application from the mockup of the screens. Thus, it is hoped that the model can contribute to the prevention of fish fraud, supporting society, industry, and government.

\section{Acknowledgments}

This work is partially supported by FCT/MCTES through national funds and when applicable co-funded EU funds under the project UIDB/50008/2020; and 
by Brazilian National Council for Scientific and Technological Development (CNPq) via Grant No. 309335/2017-5.

\section{Declarations}

Funding: This research received no external funding.

Conflict of interest: The authors declare no conflict of interest

Data availability: The authors used own data and own coding.

\section{References}

1. K. Böhme, P. Calo-Mata, J. Barros-Velázquez, I. Ortea, Recent applications of omicsbased technologies to main topics in food authentication, TrAC Trends in Analytical Chemistry 110 (2019) 221-232. doi:10.1016/j.trac.2018.11.005

2. W. Song, N. Jiang, H. Wang, J. Vincent, Use of smartphone videos and pattern recognition for food authentication, Sensors and Actuators B: Chemical 304 (2020) 127247. doi: 10.1016/j.snb.2019.127247

3. W. N. Probst, How emerging data technologies can increase trust and transparency in fisheries, ICES Journal of Marine Science 77 (4) (2020) 1286-1294. doi:10.1093/ icesjms/fsz036

4. S. A. Vo, J. Scanlan, P. Turner, R. Ollington, Convolutional neural networks for individual identification in the southern rock lobster supply chain, Food Control 118 (2020) 107419 doi:10.1016/j.foodcont.2020.107419

5. F. Rossi, A. Benso, S. Di Carlo, et al., FishAPP: A mobile App to detect fish falsification through image processing and machine learning techniques, in: IEEE International Conference on Automation, Quality and Testing, Robotics, IEEE, Cluj-Napoca, Romania, 2016, pp. 1-6. doi:10.1109/AQTR.2016.7501348.

6. H. Masuda, T. Jukei, T. Hasegawa, Fish species identification using a CNN-based multimodal learning method, in: 2nd International Conference on Image, Video and Signal Processing, ACM, Jakarta, Indonesia, 2020, pp. 15-19. doi:10.1145/3388818.3389164

7. P. Hridayami, I. K. Putra, K. S. Wibawa, Fish species recognition using VGG16 deep convolutional neural network, Journal of Computing Science and Engineering 13 (3) (2019) 124-130. doi:10.5626/JCSE.2019.13.3.124

8. L. Cui, S. Yang, F. Chen, et al., A survey on application of machine learning for internet of things, International Journal of Machine Learning and Cybernetics 9 (8) (2018) 1399-1417. doi:10.1007/s13042-018-0834-5

9. J. Zhang, D. Tao, Empowering things with intelligence: A survey of the progress, challenges, and opportunities in artificial intelligence of things, IEEE Internet of Things Journal (in press) (2020). doi:10.1109/JIOT.2020.3039359

10. Y. Ma, K. Ping, C. Wu, et al., Artificial intelligence powered internet of things and smart public service, Library Hi Tech 38 (2019) 165-179. doi:10.1108/LHT-12-2017-0274

11. L. Zhou, C. Zhang, F. Liu, Z. Qiu, Y. He, Application of deep learning in food: A review, Comprehensive Reviews in Food Science and Food Safety 18 (6) (2019) 1793-1811. doi:10.1111/1541-4337.12492

12. J. J. Rachel, P. Varalakshmi, Classification of breeding fish using deep learning from the captured video, in: 11th International Conference on Advanced Computing, IEEE, Chennai, India, 2019, pp. 48-55. doi:10.1109/ICoAC48765.2019.246815

13. K. Sanghvi, A. Aralkar, S. Sanghvi, I. Saha, Fauna image classification using convolutional neural network, International Journal of Future Generation Communication and Networking 13 (1) (2020) 08-16.

14. F. Liang, AI-powered digital media platform and its applications, in: Conference on Artificial Intelligence and Healthcare, ACM, Taiyuan, China, 2020, pp. 121-126. doi: $10.1145 / 3433996.3434018$ 
15. I. H. Sarker, M. M. Hoque, M. K. Uddin, T. Alsanoosy, Mobile data science and intelligent apps: Concepts, ai-based modeling and research directions, Mobile Networks and Applications (2020) 1-19doi:10.1007/s11036-020-01650-z

16. D. Wang, M. Zhang, Z. Li, et al., Modulation format recognition and OSNR estimation using cnn-based deep learning, IEEE Photonics Technology Letters 29 (19) (2017) 1667-1670. doi:10.1109/LPT.2017.2742553

17. A. Kamilaris, F. X. Prenafeta-Boldú, Deep learning in agriculture: A survey, Computers and Electronics in Agriculture 147 (2018) 70-90. doi:10.1016/j.compag.2018.02.016

18. H. Qin, X. Li, J. Liang, et al., DeepFish: Accurate underwater live fish recognition with a deep architecture, Neurocomputing 187 (2016) 49-58. doi:0.1016/j.neucom.2015.10 122

19. H. T. Rauf, M. I. Lali, S. Zahoor, et al., Visual features based automated identification of fish species using deep convolutional neural networks, Computers and Electronics in Agriculture 167 (2019) 105075. doi:10.1016/j.compag.2019.105075

20. A. Khan, A. Sohail, U. Zahoora, A. S. Qureshi, A survey of the recent architectures of deep convolutional neural networks, Artificial Intelligence Review (2020) 1-62 doi: 10.1007/s10462-020-09825-6

21. K. Simonyan, A. Zisserman, Very deep convolutional networks for large-scale image recognition, arXiv preprint arXiv:1409.1556 (2014).

22. C. Szegedy, V. Vanhoucke, S. Ioffe, J. Shlens, Z. Wojna, Rethinking the inception architecture for computer vision, in: IEEE Conference on Computer Vision and Pattern Recognition, IEEE, Las Vegas, NV, USA, 2016, pp. 2818-2826. doi:10.1109/CVPR. 2016 308

23. G. Huang, Z. Liu, L. Van Der Maaten, K. Q. Weinberger, Densely connected convolutional networks, in: IEEE Conference on Computer Vision and Pattern Recognition, IEEE, Honolulu, HI, USA, 2017, pp. 4700-4708. doi:10.1109/CVPR.2017.243.

24. A. G. Howard, M. Zhu, B. Chen, D. Kalenichenko, W. Wang, T. Weyand, M. Andreetto, H. Adam, Mobilenets: Efficient convolutional neural networks for mobile vision applications, arXiv preprint arXiv:1704.04861 (2017).

25. B. Khasoggi, E. Ermatita, S. Sahmin, Efficient mobilenet architecture as image recognition on mobile and embedded devices, Indonesian Journal of Electrical Engineering and Computer Science 16 (2019) 389. doi:10.11591/ijeecs.v16.i1.pp389-394

26. F. Chollet, Xception: Deep learning with depthwise separable convolutions, in: IEEE Conference on Computer Vision and Pattern Recognition, IEEE, Honolulu, HI, USA, 2017, pp. 1251-1258. doi:10.1109/CVPR.2017.195

27. A. A. Santos, W. N. Gonçalves, Improving pantanal fish species recognition through taxonomic ranks in convolutional neural networks, Ecological Informatics 53 (2019) 100977. doi:10.1016/j.ecoinf.2019.100977. 